\title{
Item Analysis di una prova di lettura a scelta multipla della certificazione di italiano per stranieri CILS (livello B1; sessione estiva 2012)
}

\section{Item Analysis of a multiple-choice reading test in the Italian certification for foreign speakers CILS (level B1; summer session 2012)}

Paolo Torresan

Università Ca' Foscari, Venezia, Veneto, Italia.

piroclastico@gmail.com

\begin{abstract}
Nell'articolo presentiamo un'analisi degli item di una prova di lettura a scelta multipla di livello B1 della certificazione CILS (Università per Stranieri di Siena). L'indagine si muove da una prima ricognizione del testo su cui si basa la prova, con uno studio delle modifiche cui è andata soggetta per mano dell'item writer, per poi ragionare sull'analisi di ogni singolo item, grazie ai dati emersi dalla somministrazione della prova a 161 studenti di italiano di livello corrispondente sparsi per il pianeta. Dalla nostra ricerca si evince che si danno un item ambiguo (\# 1), per via della presenza di due chiavi, e un item di difficile risoluzione, per via della mancanza di informazioni utili per desumere il significato del vocabolo cui si riferisce (\# 4).
\end{abstract}

Parole chiave: didattica dell'italiano come lingua straniera; Item Analysis; testing; lettura; test a scelta multipla.

Abstract: In this article we present an analysis of items in a reading multiple-choice test, B1 level, of the CILS certification (Università per Stranieri di Siena). The research starts with a preliminary recognition of the text on which the test is based, with a study of the modifications it has undergone by the item writer's hand, and proceeds to reason about 
the analysis of every single item, using data from the ministration of the test to 161 students of Italian in the corresponding level, from all over the planet. From our research it emerges that the test presents an ambiguous item (\# 1), with two keys, and a difficult item, without enough information to make clear the meaning of the word it refers to (\# 4).

Keywords: teaching Italian as a foreign language; testing; Item Analysis; reading; MCQ tests.

Recebido em 13 de maio de 2014. Aprovado em 04 de outubro de 2014.

\section{La certificazione CILS}

La certificazione CILS, rilasciata dall'Università per Stranieri di Siena, rientra, assieme alla certificazione CELI, elaborata dall'Università per Stranieri di Perugia, alla certificazione PLIDA, elaborata dalla società Dante Alighieri, e alla certificazione IT, dell'Università Roma Tre, nel progetto CLIQ, Certificazione Lingua Italiana di Qualità, siglato tra il Ministero degli Affari Esteri e i rappresentanti dei vari enti certificatori, per definire standard comuni di valutazione.

La certificazione CILS, in particolare

è stata progettata e realizzata all'interno del Centro CILS, un centro di Ricerca e di Servizi che ha una funzione autonoma entro l'Università di Siena, ed è indipendente dai Centri che erogano servizi di formazione linguistica. La natura stessa del Centro, responsabile e autonomo a livello teorico e operativo, ha fatto sì che all'interno di esso si potesse sviluppare una attività di ricerca, sia per quanto concerne i modelli teorico-metodologici di riferimento per la descrizione e l'articolazione in livelli della competenza linguistico-comunicativa, sia per le scelte relative alla sua verifica, valutazione e certificazione. (BARNI et al., 2009, p. 10)

Il nostro contributo intende aprire un dibattito in Italia sulla struttura delle prove delle certificazioni italiane. Riteniamo uno sguardo esterno possa costituire: 
- un motivo di approfondimento di prassi consolidate, suggerendo, laddove possibile, eventuali miglioramenti nel design e nei percorsi di validazione;

- una condivisione dei risultati dell'item analysis con insegnanti, studenti e amministratori, affinché si possano generare nuove esperienze di validazione.

L'appello a uno sguardo esterno nella validazione delle prove viene invocato da ALDERSON et al., 1995, p. 63:

It is absolutely crucial in all test development, for whatever purpose, at whatever level of learner ability and however trivial the consequences of failure on test might be, that some person or persons other than the individual item writer look closely at each item, respond to the item as a student would, reflect upon what abilities are required for successful completion of the item/task, and then compare what he or she thinks the items is testing with what the item writer claims it test.

Nel passo citato l'auspicio è che altri, rispetto al singolo item writer, eseguano un'analisi critica delle prove, offrendo un nuovo punto di vista; ciò non esclude, appunto, l'intervento di esperti esterni al centro nel quale la prova è stata confezionata.

La certificazione CILS prevede un rigoroso protocollo a garanzia dell'affidabilità e della validità delle prove (BARNI et al., 2009, 19):

Allo scopo di garantire l'affidabilità delle prove il Centro CILS ha adottato delle procedure per la garanzia dell'affidabilità delle prove che si attuano in due fasi: prima e dopo la somministrazione del test. Per le prove chiuse si ricorre all'item analysis [...]. Dopo la prima stesura delle prove viene realizzato un pre-testing per verificare la validità e l'affidabilità degli item. Il pre-testing è effettuato su un campione di individui con competenze adeguate al livello per cui la prova è costruita. Le risposte ottenute sono sottoposte ad analisi statistiche, per rilevare gli item troppo facili o troppo difficili; per individuare se ci sono distrattori, cioè le risposte non esatte proposte accanto alle risposte esatte nelle prove oggettive, che hanno una percentuale di scelta troppo bassa o troppo alta; 
per verificare se gli item sono in grado di discriminare $\mathrm{i}$ candidati più competenti da quelli meno competenti, e per poter effettuare le opportune tarature delle prove.

Dopo la somministrazione del test, l'analisi degli item viene ripetuta sulle risposte date dai candidati che hanno effettivamente sostenuto l'esame CILS.

Quella che proponiamo, nel nostro caso, è un'Item Analysis esterna che possa, in linea teorica, confermare che le chiavi siano definite con rigore (non ambigue) e i distrattori siano efficaci.

\section{La prova oggetto di studio}

Il test a scelta multipla oggetto di analisi si compone di sette item, con quattro distrattori ciascuno, riferiti a un brano tratto dall'attualità soggetto a rimaneggiamento.

\subsection{L'input}

Il testo originale (TO) è stato tratto da un articolo apparso sul sito di una catena di supermercati.

Nella presentazione affiancata della fonte autentica e del testo modificato (d'ora in poi TM), sono messe in risalto le modifiche operate dal certificatore.

TESTO ORIGINALE

barrato: parte soggetta a espunzione

con fondino: parte soggetta a manipolazione significativa

[PTO]: problemi nel testo originale

TESTO MODIFICATO CILS

sottolineato: inserzioni

con fondino: parte soggetta a manipolazione significativa

[PTM]: problemi nel testo modificato

riquadrettato informazioni equivalenti su porzioni distinte dei testi 


\section{Testo Originale}

http://www.coopfirenze.it/informatori/ notizie/spesa-elettronica-a-102anni-11984

(C) Laura D'Ettole, Sito della UniCoop Firenze, 27 marzo 2012

\section{Spesa elettronica a $\mathbf{1 0 2}$ anni}

Annita ha voluto la carta elettronica per pagare la spesa «Mi hanno voluto tutti tanto bene e tutti mi dicevano: tu non devimorire. Cosi ho fatto!n. Promessa mantenuta. Annita, elasse 1910, frorentina di naseita, [PTO1 $\rightarrow$ ] ha 102 anni ed è socia, più anziana di Unicoop Firenze stessa, iseritta da sempre.

Per Annita gli informatici di Integra, la società che gestisce la carta socio abilitata al pagamento elettronico della spesa, hanno dovuto fare gli straordinari: infatti nel modulo/contratto erano previste due sole caselle per l'età. "Chi può chiedere una carta elettronica a 100 anni?" si erano probabilmente chiesti. Annita! Eisistemi erano andati in tilt, ma poi sono stati riadattati a questo fenomeno di longevità. Annita è iscritta al libro soci della Coop dal 1971, aneor prima dell'anno ehe fu pietra miliare, quel 1973 in eui la fusione fra le due cooperative di Firenze e di Empoli dette vita alla maggiore eoop di eonsumo itatiana.

«Io ho sempre fatto la spesa alla Coop. Ancora oggi ci vado due volte la settimana». Da sola? "Certo, da sola e a piedi. Qualche volta mi faccio accompagnare, ma poi faccio tutto da sola perché sono incontentabile. Mangio di tutto. Perfino la panna con $i$ cialdoni...». Annita, to diee senza modestia, è una grande cuoca perché ha imparato da suo padre. «Il babbo era il primo cuoco di Cencio di Borgo San Lorenzo... Come, non lo eonosee? E roba di prima categoria». Annita arriva al negozio Coop dove tutti la conoscono

\section{Testo Modificato CILS (CON CHIAVI INSERITE)}

Spesa elettronica a 102 anni

Anita, un vulcano di attività, ha voluto la carta elettronica per pagarsi la spesa. Anita è nata a Perugia, ha 102 anni ed è socia da sempre del supermercato Super a Perugia. Per Anita gli informatici di Integra, la società che gestisce la carta socio per fare i pagamenti elettronici della spesa, hanno dovuto fare gli straordinari: infatti nel contratto c'erano solo due caselle per l'età. "Chi può chiedere la carta elettronica a 100 anni?"- si sono probabilmente detti gli informatici! E invece Anita l'ha voluta e gli informatici hanno riadattato il sistema informatico.

Anita è iscritta al libro dei soci del supermercato Super dal 1971. "Io ho sempre fatto la spesa alla Super", dice Anita, "ancora oggi ci vado due volte alla settimana. Da sola e a piedi". Al supermercato Super tutti la conoscono e la vogliono aiutare, ma lei rifiuta l'offerta con molta gentilezza. E sempre vivace. Saluta e sorride a tutti. Ora che ha la sua carta socio non ha nemmeno bisogno di contare le monete!

"Qualche volta mi faccio accompagnare, ma poi faccio tutto da sola perché sono incontentabile", racconta Anita. "Mangio di tutto, perfino la panna con i cialdoni, l'adoro". Anita è una grande cuoca perché ha imparato da suo padre che era cuoco a Ponte San Giovanni, dove gestiva una trattoria di prima categoria. Anita è nata a maggio nel 1910. Ha fatto di tutto per mantenersi. "Il lavoro è uno dei miei ricordi più belli: ho iniziato 17 
e la vogliono aiutare. Lei declina gli aiuti con gentilezza. È sempre vivace. Saluta, sorride a tutti. Ora ha anche una fiammante carta socio Spesa In, e quindi non ha bisogno di contare gli spiccioli... tha bella comodità.

E nata a maggio nel 1910, appena in tempo per vedere gli ultimi seorei della belle époque. Aveva due anni quando, nel 1912, affondò il Titanic. Poi, nulla fu eome prima: "Il Piave mormorom, eanticehia. Ha passato due guterre mondiali, una dittatura kterribite paurosa, più della guerra». Ha fatto di tutto per mantenersi: «Il lavoro è uno dei miei ricordi più belli: ho iniziato a 17 anni. Ho fatto il "plissé" per gli abiti di moda, l'infermiera, ta macellaia. Ho smesso di lavorare a 75 anni». Musiea per le oreechie degli enti previdenziali. Annita ha vissuto una vita complieata, per 94 anni insieme alla sorella Alina, che ora none'è pitù. Non si è mai voluta sposare, ma ha una folla di nipoti, pronipoti di primo e secondo grado. E un gatto, Ehiees, "rehe sembra un cane per tutti $i$ baciniche mi dà mi fa tanta compagnia. La sera, prima di andare a letto, se non gli ho dato la buona notte [PTO2 $\rightarrow$ ] protesta e mi tocca alzarmi di nuovo-... Senta, ha altro da chiedermi? Ho furia, devo andare a farmi da mangiare. Mi richiami eh, mi richiami!n. Tantiauguri, signora Annita. anni, ho fatto la sarta, ma non ero molto abile, l'infermiera, la commessa in un negozio di abbigliamento. Ho smesso di lavorare a 75 anni". Anita ha vissuto per molti anni vicino alla sorella, non si è mai voluta sposare, ma ha molti nipoti. Non ama la televisione. "Avevo un cane", dice Anita, "ma era troppo impegnativo portarlo fuori tre volte al giorno". Ora ha un gatto, che le fa tanta compagnia. "La sera, prima di andare a letto, se non gli ho dato la buona notte $[$ PTM1 $\rightarrow$ ] protesta e devo alzarmi di nuovo".

Le criticità riscontrate nei testi sono poco significative (eccetto PTO1, che è stata comunque emendata dal certificatore); quelle del TM non incidono affatto sulla perfomance dei candidati. Le alterazioni al TO sono avvenute, quindi, nel rispetto dei significati, nonché della coesione e della coerenza del testo di partenza. 


\begin{tabular}{|c|c|c|c|}
\hline & Testo Originale & $\begin{array}{l}\text { Emendamento } \\
\text { Suggerito }\end{array}$ & Commento \\
\hline PTO1 & $\begin{array}{l}{[\rightarrow] \text { ha } 102 \text { anni ed è }} \\
\text { socia, più anziana di } \\
\text { Unicoop Firenze stessa, } \\
\text { iscritta da sempre }\end{array}$ & $\begin{array}{l}\text { ha } 102 \text { anni ed è }[\rightarrow] \\
\text { la socia più anziana di } \\
\text { Unicoop Firenze }[\rightarrow] \\
\text { [stessa], iscritta da } \\
\text { sempre }\end{array}$ & $\begin{array}{l}\text { La frase è scoordinata. } \\
\mathrm{E} \text { n e c s s a ri o un } \\
\text { rimaneggiamento. }\end{array}$ \\
\hline PTO2 & $\begin{array}{l}\text { La sera, prima di andare } \\
\text { a letto, se non gli ho } \\
\text { dato la buona notte }[\rightarrow] \\
\text { protesta }\end{array}$ & $\begin{array}{l}\text { La sera, prima di } \\
\text { andare a letto, se non } \\
\text { gli ho dato la buona } \\
\text { notte }[\rightarrow] \text {, protesta }\end{array}$ & $\begin{array}{l}\text { Va inserita una virgola, } \\
\text { a chiusura dell'inciso. }\end{array}$ \\
\hline
\end{tabular}

\begin{tabular}{|c|c|c|c|}
\hline & $\begin{array}{c}\text { Testo Modificato } \\
\text { CILS }\end{array}$ & \multicolumn{1}{|c|}{$\begin{array}{c}\text { Emendamento } \\
\text { Suggerito }\end{array}$} & Commento \\
\hline PTM1 & $\begin{array}{l}\text { La sera, prima di } \\
\text { andare a letto, se non } \\
\text { gli ho dato la buona } \\
\text { notte }[\rightarrow] \text { protesta }\end{array}$ & $\begin{array}{l}\text { La sera, prima di andare } \\
\text { a letto, se non gli ho } \\
\text { dato la buona notte }[\rightarrow] \\
\text {, protesta }\end{array}$ & $\begin{array}{l}\text { Va inserita una virgola, } \\
\text { a chiusura dell'inciso } \\
\text { (cfr. PTO2). }\end{array}$ \\
\hline
\end{tabular}

\subsection{Gli item}

Abbiamo somministrato la prova a 161 studenti di livello B1, avvalendoci della disponibilità di colleghi che operano sia in Italia (in prevalenza nelle scuole private) che all' estero (in prevalenza negli Istituti di Cultura e nelle università).

Nella tabella che segue riportiamo i facility value di ciascun item (d'ora in poi $\mathrm{FV})^{1}$.

\footnotetext{
${ }^{1}$ Il facility value (FV) corrisponde con la percentuale di studenti che rispondono correttamente ai singoli item.

Si ritiene che un item, in un proficiency test qual è il CILS, 'funzioni' nella misura in cui discrimina gli esaminandi in base alla competenza, vale a dire distingue i candidati competenti da quelli che non lo sono, in riferimento al livello per il quale il test è stato confezionato. Se l'item risulta troppo facile (e quindi non discrimina a sufficienza) o al contrario se risulta troppo difficile, la prova è in disequilibrio rispetto al livello al quale viene riferita (McNAMARA, 2000, pp. 60-61): "Where the text purpose is to make distinctions between candidates, to spread them out in terms of their performance on the texts, the items should be neither too easy nor too difficult. If the items are too easy, then people with differing levels of ability or knowledge will all get them right,
} 
Item \# 1

\begin{tabular}{|c|l|c|c|}
\hline \multicolumn{2}{|c|}{} & Frequenza & Percentuale \\
\hline \multirow{4}{*}{ Validi } & Errato & 23 & 14,3 \\
& Corretto & 138 & 85,7 \\
\cline { 2 - 3 } & Totale & 161 & 100,0 \\
\hline
\end{tabular}

Item \# 2

\begin{tabular}{|c|l|c|c|}
\hline \multicolumn{2}{|c|}{} & Frequenza & Percentuale \\
\hline \multirow{4}{*}{ Validi } & Errato & 54 & 33,5 \\
& Corretto & 107 & 66,5 \\
\cline { 2 - 3 } & Totale & 161 & 100,0 \\
\hline
\end{tabular}

and the differences in ability or knowledge will not be revealed by the item. Similarly if the items are too hard, then able and less able candidates alike will get them wrong, and the item won't help us in distinguishing between them".

Quale FV considerare per stabilire l'appropriatezza di un item rispetto al livello dichiarato?

Lo stesso autore dichiara (2000, p. 67): "Ideal item facility [i.e. Facility Value, ndt.] is 0.5 [i.e. 50\%, ndt.] but of course it is hard to hit this target exactly, and a range of item facilities from 0.33 [i.e. 33\%, ndt.] to 0.67 [i.e. 67\%, ndt.] is usually accepted". La posizione di Green riconosce margini più ampi (2013, pp. 26-27): "The most useful information about a test taker's proficiency comes from facility values which are around 50 per cent (see POPHAM 2000) as this value suggests that the item might be discriminating between the test takers (though it is not always the case); 40 to 60 per cent (see BACHMAN 2004), which are the values many test developers use when making initial decisions about whether an item is working or not.

Facility values between 20 and 80 per cent can also provide useful information provided the items still discriminate and contribute to the test's internal consistency [...].

Facility values of below 20 per cent and above 80 per cent in a proficiency test suggest that most of the test population is either answering the item incorrectly or correctly respectively, which reduces the chance of gaining useful information about the test takers and/or the items". 
Item \# 3

\begin{tabular}{|c|l|c|c|}
\hline \multicolumn{2}{|c|}{} & Frequenza & Percentuale \\
\hline \multirow{4}{*}{ Validi } & Errato & 24 & 14,9 \\
\cline { 2 - 3 } & Corretto & 137 & 85,1 \\
\cline { 2 - 3 } & Totale & 161 & 100,0 \\
\hline
\end{tabular}

Item \# 4

\begin{tabular}{|c|l|c|c|}
\hline \multicolumn{2}{|c|}{} & Frequenza & Percentuale \\
\hline \multirow{4}{*}{ Validi } & Errato & 105 & 65,2 \\
\cline { 2 - 3 } & Corretto & 56 & 34,8 \\
\cline { 2 - 3 } & Totale & 161 & 100,0 \\
\hline
\end{tabular}

Item \# 5

\begin{tabular}{|c|l|c|c|}
\hline \multicolumn{2}{|c|}{} & Frequenza & Percentuale \\
\hline \multirow{4}{*}{ Validi } & Errato & 27 & 16,8 \\
& Corretto & 134 & 83,2 \\
& Totale & 161 & 100,0 \\
\hline
\end{tabular}

Item \# 6

\begin{tabular}{|c|l|c|c|}
\hline \multicolumn{2}{|c|}{} & Frequenza & Percentuale \\
\hline \multirow{4}{*}{ Validi } & Errato & 39 & 24,2 \\
\cline { 2 - 3 } & Corretto & 122 & 75,8 \\
\cline { 2 - 3 } & Totale & 161 & 100,0 \\
\hline
\end{tabular}

Item \# 7

\begin{tabular}{|c|l|c|c|}
\hline \multicolumn{2}{|c|}{} & Frequenza & Percentuale \\
\hline \multirow{4}{*}{ Validi } & Errato & 16 & 9,9 \\
\cline { 2 - 3 } & Corretto & 145 & 90,1 \\
\cline { 2 - 3 } & Totale & 161 & 100,0 \\
\hline
\end{tabular}

Il seguente istogramma (fig. 1) illustra la distribuzione dei punteggi complessivi raggiunti dal campione. La maggior parte degli studenti di italiano a cui il test è stato somministrato (55) ha risposto correttamente a 6 item su 7 (la colonna in riferimento al \# 6 sulle ascisse è, in effetti, la maggiore). Si tratta di un profilo con disallineamento 
negativo (negatively skewed): il test è, cioè, relativamente facile per il campione di candidati a cui è stato somministrato.

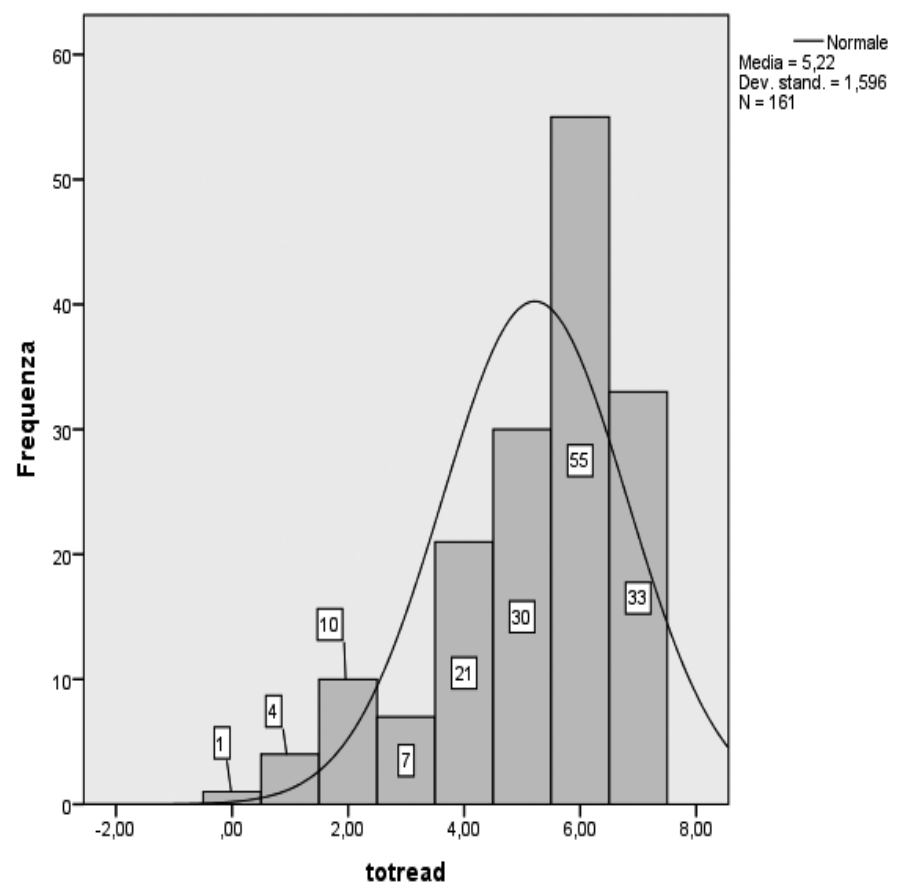

Fig. 1. Punteggi dei candidati alla prova di lettura a scelta multipla Certificazione CILS, Sessione giugno 2012, livello B1

Come si evince dalla tabella che segue, l'item \# 4 presenta un elevato numero di risposte nulle (è il più difficile, con un FV pari a 34,8: solo uno studente su tre ha indovinato la risposta).

\begin{tabular}{|c|l|c|c|c|c|c|c|c|}
\hline \multicolumn{2}{|c|}{} & Item \# 1 & Item \# 2 & Item \# 3 & Item \# 4 & Item \# 5 & Item \# 6 & Item \# 7 \\
\hline \multirow{2}{*}{$\mathrm{N}$} & Validi & 159 & 156 & 158 & 153 & 158 & 157 & 158 \\
\cline { 2 - 9 } & Mancanti & 2 & 5 & 3 & 8 & 3 & 4 & 3 \\
\hline
\end{tabular}


Consideriamo le specificità di ciascun item.

\section{Item \# 1}

È un item facile: vi è una esatta corrispondenza tra la chiave e un'informazione presentata nella parte centrale testo:

chiave: [Anita è un'anziana signora] molto vivace TM: $\quad$ è sempre vivace

Con ogni probabilità l'item writer si è però riferito all'incipit del testo (dato che si tratta del primo item), dove si dice che Anita è "un vulcano di attività".

A fronte di tanta ridondanza, c'è da riconoscere tuttavia che il distrattore d) (il quale ha raccolto un numero discreto di consensi, circa il 12\%) può trarre in inganno. Il distrattore d) recita: [Anita è un 'anziana signora] molto socievole. Sempre nella parte centrale del testo si legge: "Al supermercato Super tutti la conoscono"; poco sotto: "Saluta e sorride a tutti". La socievolezza di Anita è, quindi, desumibile dal testo. Eppure "There should be one option that is clearly correct, not two or more that are partially correct" (CARR, 2011, p. 91).

La situazione di questo item è quindi del tutto singolare; da un lato abbiamo nel testo una resa letterale della chiave prevista dal certificatore, che lo configura come un item estremamente accessibile, dall'altro si constata la presenza di una doppia chiave che incide negativamente sul FV.

\section{Anita è un'anziana signora}

A) parecchio silenziosa.

B) troppo irritabile.

C) molto vivace.

D) estremamente socievole.

\begin{tabular}{|c|c|c|c|c|c|}
\hline \multicolumn{2}{|c|}{} & Frequenza & Percentuale & $\begin{array}{c}\text { Percentuale } \\
\text { valida }\end{array}$ & $\begin{array}{c}\text { Percentuale } \\
\text { cumulata }\end{array}$ \\
\hline \multirow{4}{*}{ Validi } & B & 2 & 1,2 & 1,3 & 1,3 \\
\cline { 2 - 6 } & $\mathrm{C}=$ Chiave & 138 & 85,7 & 86,8 & 88,1 \\
\cline { 2 - 6 } & $\mathrm{D}$ & 19 & 11,8 & 11,9 & 100,0 \\
\cline { 2 - 6 } & Totale & 159 & 98,8 & 100,0 & \\
\hline
\end{tabular}




\begin{tabular}{|l|c|c|c|c|c|}
\hline Mancanti & $\begin{array}{c}\text { Nessuna } \\
\text { risposta }\end{array}$ & 2 & 1,2 & & \\
\hline Totale & 161 & 100,0 & & \\
\hline
\end{tabular}

Item \# 2

L'item si presenta come relativamente difficile, plausibilmente data la complessità con cui sono stati formulati distrattori e chiave².

\section{La società Integra ha dovuto lavorare molto per Anita perché}

A) Anita non riusciva ad usare la carta elettronica e ha causato blocchi al sistema B) nel modulo di richiesta della carta mancava lo spazio per scrivere l'età di Anita

C) Anita ha perso la carta elettronica varie volte e la società ha dovuto fare dei duplicati.

D) la società ha ristampato il modulo con caratteri più grandi per permettere ad Anita di leggerlo.

\begin{tabular}{|c|l|c|c|c|c|}
\hline \multicolumn{2}{|c|}{} & Frequenza & Percentuale & $\begin{array}{c}\text { Percentuale } \\
\text { valida }\end{array}$ & $\begin{array}{c}\text { Percentuale } \\
\text { cumulata }\end{array}$ \\
\hline \multirow{5}{*}{ Validi } & A & 19 & 11,8 & 12,2 & 12,2 \\
\cline { 2 - 6 } & B=Chiave & 107 & 66,5 & 68,6 & 80,8 \\
\cline { 2 - 6 } & C & 19 & 11,8 & 12,2 & 92,9 \\
\cline { 2 - 6 } & D & 11 & 6,8 & 7,1 & 100,0 \\
\cline { 2 - 6 } & Totale & 156 & 96,9 & 100,0 & \\
\hline \multirow{5}{*}{ Mancanti } & $\begin{array}{l}\text { Nessuna } \\
\text { risposta }\end{array}$ & 4 & 2,5 & & \\
\cline { 2 - 7 } & $\begin{array}{l}\text { Doppia } \\
\text { risposta }\end{array}$ & 1 &, 6 & & \\
\cline { 2 - 7 } & Totale & 5 & 3,1 & & \\
\hline Totale & 161 & 100,0 & & \\
\hline
\end{tabular}

Item \# 3

L'item si presenta relativamente facile; i distrattori c) e d) hanno raccolto pochi consensi.

${ }^{2}$ Numerosi autori insistono sia opportuno, nei test a scelta multipla, scrivere item di facile lettura (cfr. THORNDIKE, HAGEN, 196933 CANGELOSI, 1990; HALADYNA, et al., 2002). 
3. Quando va a fare la spesa, Anita A) non vuole nessuno con lei.

B) chiede ai suoi vicini di accompagnarla con l'auto.

C) porta una grande borsa con le ruote

D) non sa mai cosa comprare.

\begin{tabular}{|c|l|c|c|c|c|}
\hline \multicolumn{2}{|c|}{} & Frequenza & Percentuale & $\begin{array}{c}\text { Percentuale } \\
\text { valida }\end{array}$ & $\begin{array}{c}\text { Percentuale } \\
\text { cumulata }\end{array}$ \\
\hline \multirow{5}{*}{ Validi } & A=Chiave & 137 & 85,1 & 86,7 & 86,7 \\
\cline { 2 - 6 } & $\mathrm{B}$ & 10 & 6,2 & 6,3 & 93,0 \\
\cline { 2 - 6 } & $\mathrm{C}$ & 5 & 3,1 & 3,2 & 96,2 \\
\cline { 2 - 6 } & $\mathrm{D}$ & 6 & 3,7 & 3,8 & 100,0 \\
\cline { 2 - 6 } & Totale & 158 & 98,1 & 100,0 & \\
\hline \multirow{5}{*}{ Mancanti } & $\begin{array}{l}\text { Nessuna } \\
\text { risposta }\end{array}$ & 2 & 1,2 & & \\
\cline { 2 - 7 } & $\begin{array}{l}\text { Doppia } \\
\text { risposta }\end{array}$ & 1 &, 6 & & \\
\cline { 2 - 7 } & Totale & 3 & 1,9 & & \\
\hline Totale & & 161 & 100,0 & & \\
\hline
\end{tabular}

\section{item \# 4}

L'item si presenta molto difficile. Che cosa sia "la panna con $\mathrm{i}$ cialdoni" non è chiaro nemmeno a un italiano settentrionale, che potrebbe essere perplesso tra la chiave a) e il distrattore b).

Ad uno straniero la scelta si presenta ancor più ardua, venendo meno nel co-testo ogni possibile appiglio (come potrebbero essere le risposte a domande del tipo: "Dove si comprano i «cialdoni»?"; "Come e quando si mangiano?"). Il distrattore d) supera così, in termini di scelte effettuate dai test taker, la chiave: metà del campione ha ritenuto si tratti di un tipo di pane!

Se facciamo riferimento al CEFR (COUNCIL OF EUROPE, 2001), viene detto che uno studente B1 è sì "in grado di estrapolare dal contesto il significato di una parola sconosciuta e ricostruire il significato della frase", ma "a condizione di avere familiarità con l'argomento in questione". Situazione estranea da quella che si prefigura in questo item. 
4. Anita è golosa di

A) cibi dolci.

B) gelato.

C) pasta.

D) pane casalingo.

\begin{tabular}{|c|l|c|c|c|c|}
\hline \multicolumn{2}{|c|}{} & Frequenza & Percentuale & $\begin{array}{c}\text { Percentuale } \\
\text { valida }\end{array}$ & $\begin{array}{c}\text { Percentuale } \\
\text { cumulata }\end{array}$ \\
\hline \multirow{5}{*}{ Validi } & A=Chiave & 55 & 34,2 & 35,9 & 35,9 \\
\cline { 2 - 6 } & $\mathrm{B}$ & 10 & 6,2 & 6,5 & 42,5 \\
\cline { 2 - 6 } & $\mathrm{C}$ & 10 & 6,2 & 6,5 & 49,0 \\
\cline { 2 - 6 } & $\mathrm{D}$ & 78 & 48,4 & 51,0 & 100,0 \\
\cline { 2 - 6 } & Totale & 153 & 95,0 & 100,0 & \\
\hline \multirow{5}{*}{ Mancanti } & $\begin{array}{l}\text { Nessuna } \\
\text { risposta }\end{array}$ & 7 & 4,3 & & \\
\cline { 2 - 6 } & $\begin{array}{l}\text { Doppia } \\
\text { risposta }\end{array}$ & 1 &, 6 & & \\
\cline { 2 - 7 } & Totale & 8 & 5,0 & & \\
\hline Totale & & 161 & 100,0 & & \\
\hline
\end{tabular}

Item \# 5

L'item discrimina adeguatamente; solo il distrattore d) pare essere pressoché totalmente ignorato.

\section{Anita è molto brava a}

A) cucire abiti

B) raccontare storie.

C) cucinare.

D) curare le piante.

\begin{tabular}{|l|l|l|l|l|l|}
\hline \multicolumn{2}{|c|}{} & Frequenza & Percentuale & $\begin{array}{l}\text { Percentuale } \\
\text { valida }\end{array}$ & $\begin{array}{l}\text { Percentuale } \\
\text { cumulata }\end{array}$ \\
\hline \multirow{6}{*}{ Validi } & A & 7 & 4,3 & 4,4 & 4,4 \\
\cline { 2 - 6 } & B & 15 & 9,3 & 9,5 & 13,9 \\
\cline { 2 - 6 } & C=Chiave & 134 & 83,2 & 84,8 & 98,7 \\
\cline { 2 - 6 } & D & 2 & 1,2 & 1,3 & 100,0 \\
\cline { 2 - 6 } & Totale & 158 & 98,1 & 100,0 & \\
\hline
\end{tabular}




\begin{tabular}{|l|l|l|l|l|l|}
\hline Mancanti & $\begin{array}{l}\text { Nessuna } \\
\text { risposta }\end{array}$ & 3 & 1,9 & & \\
\hline Totale & 161 & 100,0 & & \\
\hline
\end{tabular}

Item \# 6

L'item funziona adeguatamente.

6. Della sua vita Anita ricorda con piacere

A) le attività lavorative.

B) le amiche sarte.

C) la trattoria del padre.

D) la casa di Ponte San Giovanni.

\begin{tabular}{|c|l|c|c|c|c|}
\hline \multicolumn{2}{|c|}{} & Frequenza & Percentuale & $\begin{array}{c}\text { Percentuale } \\
\text { valida }\end{array}$ & $\begin{array}{c}\text { Percentuale } \\
\text { cumulata }\end{array}$ \\
\hline \multirow{5}{*}{ Validi } & A=Chiave & 122 & 75,8 & 77,7 & 77,7 \\
\cline { 2 - 6 } & $\mathrm{B}$ & 1 &, 6 &, 6 & 78,3 \\
\cline { 2 - 6 } & $\mathrm{C}$ & 20 & 12,4 & 12,7 & 91,1 \\
\cline { 2 - 6 } & $\mathrm{D}$ & 14 & 8,7 & 8,9 & 100,0 \\
\cline { 2 - 6 } & Totale & 157 & 97,5 & 100,0 & \\
\hline \multirow{5}{*}{ Mancanti } & $\begin{array}{l}\text { Nessuna } \\
\text { risposta }\end{array}$ & 3 & 1,9 & & \\
\cline { 2 - 7 } & $\begin{array}{l}\text { Doppia } \\
\text { risposta }\end{array}$ & 1 &, 6 & & \\
\cline { 2 - 7 } & Totale & 4 & 2,5 & & \\
\hline Totale & & 161 & 100,0 & & \\
\hline
\end{tabular}

\section{Item \# 7}

L'item si rivela estremamente facile. I distrattori a) e c) hanno raccolto pochissimi consensi.
A) telefona ai suoi nipotini.
B) porta fuori il cane
C) guarda un po' la TV.
D) saluta il suo gatto.

7. Tutte le sere, prima di dormire, Anita 


\begin{tabular}{|c|l|c|c|c|c|}
\hline \multicolumn{2}{|c|}{} & Frequenza & Percentuale & $\begin{array}{c}\text { Percentuale } \\
\text { valida }\end{array}$ & $\begin{array}{c}\text { Percentuale } \\
\text { cumulata }\end{array}$ \\
\hline \multirow{3}{*}{ Validi } & A & 3 & 1,9 & 1,9 & 1,9 \\
\cline { 2 - 6 } & $\mathrm{B}$ & 9 & 5,6 & 5,7 & 7,6 \\
\cline { 2 - 6 } & $\mathrm{C}$ & 1 &, 6 &, 6 & 8,2 \\
\cline { 2 - 6 } & $\mathrm{D}=$ Chiave & 145 & 90,1 & 91,8 & 100,0 \\
\cline { 2 - 6 } & Totale & 158 & 98,1 & 100,0 & \\
\hline \multirow{2}{*}{ Mancanti } & $\begin{array}{l}\text { Nessuna } \\
\text { risposta }\end{array}$ & 3 & 1,9 & & \\
\hline \multicolumn{2}{|l|}{ Totale } & 161 & 100,0 & & \\
\hline
\end{tabular}

\section{Bilancio conclusivo}

La prova pare essere calibrata sulle competenze di un candidato di livello B1.

Le alterazioni del TO non hanno prodotto criticità significative.

A nostro avviso comunque la prova avrebbe dovuto essere rivista in corrispondenza a due item:

- \# 1, data la compresenza di due chiavi;

- $\quad$ \#4, di difficile risoluzione per via della mancanza di informazioni utili per poter desumere il significato del vocabolo cui si riferisce.

\section{Riferimenti}

ALDERSON, C.; CLAPHAM, C.; WALL, D. Language test construction and evaluation, Cambridge: CUP, 1995.

BACHMAN L. F. Fundamental considerations in language testing. Oxford: OUP, 1990.

BARNI, M.; BANDINI, A.; SPRUGNOLI, L.; LUCARELLI, S.; SCAGLIOSO A. M. M.; STRAMBI, B.; FUSI, C.; ARRUFFOLI A. M. Linee guida CILS. Certificazione di italiano come LS Università per stranieri di Siena. Perugia: Guerra, 2009.

CANGELOSI, J. S. Designing tests for evaluating student achievement. New York: Longman, 1990.

COUNCIL OF EUROPE. The common European framework of reference for languages: learning, teaching, assessment. Cambridge: CUP, 2001. 
CARR, N. T. Designing and analyzing language tests. Oxford: OUP, 2011. GREEN, Rita. Statistical analyses for language teachers. Basingtoke: Palgrave MacMillan, 2013.

HALADYNA, T. M.; DOWNING, S. M., RODRIGUEZ, M. C. A review of multiple-choice item-writing guidelines for classroom assessment. In: Applied measurement in education, 15, 3, 309-334, 2002.

MCNAMARA, Tim. Language testing. Oxford: OUP, 2000.

POPHAM, James W. Modern educational measurement. Boston: Allyn \& Bacon, 2000.

THORNDIKE, R. L.; HAGEN, E. Measurement and evaluation in psychology and education. New York: John Wiley and Sons, $1969^{3}$.

\section{Riferimento sitografico}

Certificazione CILS, Prove B1, sessione giugno 2012

$<$ http://cils.unistrasi.it/89/197/Prove_Liv._B1.htm> 
